\title{
Next Nearest Neighbors Effects on Berry Curvature of Graphene
}

\author{
T. Farajollahpour, A.H. Rezvani*, M.R. Khodarahmi and M. Arasteh \\ Department of Physics, Faculty of Science, P.O. Box 16575-347, I.H.U Tehran, Iran
}

(Received November 1, 2011; in final form January 11, 2012)

\begin{abstract}
In this paper energy bands and Berry curvature of graphene was studied. Desired Hamiltonian regarding the next-nearest neighbors was obtained by tight-binding model. By using the second quantization approach, the transformation matrix is calculated and the Hamiltonian of system is diagonalized. With this Hamiltonian, the band structure and wave function can be calculated. By using calculated wave function the Berry connection and Berry curvature of our system are calculated. Our results are exactly consistent with previous methods and also the Berry curvature throughout the Brillouin zone get zero.
\end{abstract}

PACS: 73.22.Pr, 81.05.ue

\section{Introduction}

Graphene produces a lot of excitement in the scientific world and has generated intense interest since it was firstly discovered in 2004 [1]. Carbon atoms are strongly bonded by $s p^{2}$ hybridization and form one-atom thick hexagonal network, which is called graphene and can be seen with an ordinary optical microscope [1-3]. It is well known that graphene is a zero-gap semiconductor and conducts electricity better than silicon [4]. Tight-binding (TB) approach is widely used to investigate physical properties of graphene and carbon nanotubes [5-10]. The first TB description of graphene was given by Wallace, who only took the nearest and next-nearest neighbor atoms into account [10]. After that, Saito et al. [11] considered the nonfinite overlap between the basic function, but included only the nearest neighbors within the graphene sheet.

On the other hand, there is a Berry phase concept in solid state physics. In 1984, Berry wrote a paper that has generated immense interests throughout the different fields of physics including quantum chemistry [12] so the Berry curvature of graphene throughout the Brillouin zone was calculated.

\section{Graphene energy band structure by nearest and next nearest neighbors}

Graphene is made out of carbon atoms arranged in hexagonal structure, as shown in Fig. 1. The structure can be seen as a triangular lattice with a basis of two atoms per unit cell. The lattice vectors can be written as $a_{1}=\frac{a}{2}(3, \sqrt{3}), a_{2}=\frac{a}{2}(3,-\sqrt{3})$ where $a=1.42 \AA$ is the carbon-carbon distance. The reciprocal-lattice vectors are given by $b_{1}=\frac{2 \pi}{3 a}(1, \sqrt{3}), b_{2}=\frac{2 \pi}{3 a}(1,-\sqrt{3})$.

* corresponding author; e-mail: arezvani@ihu.ac.ir
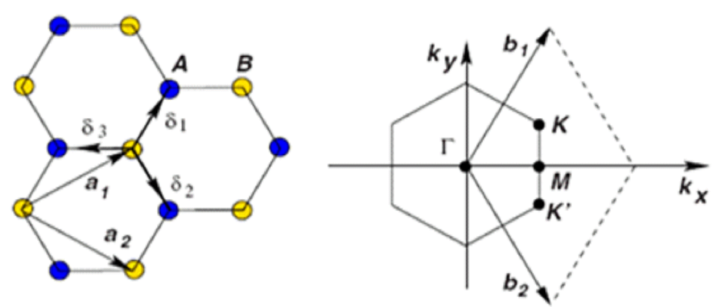

Fig. 1. Honeycomb lattice and Brillouin zone [13].

Of particular importance for the physics of graphene are the two points $K$ and $K^{\prime}$, at the corners of the graphene Brillouin zone (BZ). These are named the Dirac points. Their positions in momentum space are given by $K=\left(\frac{2 \pi}{3 a}, \frac{2 \pi}{3 \sqrt{3}}\right), K^{\prime}=\left(\frac{2 \pi}{3 a}, \frac{-2 \pi}{3 \sqrt{3} a}\right)$. Three nearest-neighbor vectors in real space are given by $\delta_{1}=$ $\frac{a}{2}(1, \sqrt{3}) \delta_{2}=\frac{a}{2}(1,-\sqrt{3}) \delta_{3}=-a(1.0)$, while the six second-nearest neighbors are located at $\delta_{1}^{\prime}= \pm a_{1}, \delta_{2}^{\prime}=$ $\pm a_{2}, \delta_{3}^{\prime}= \pm\left(a_{2}-a_{1}\right)$.

The tight-binding Hamiltonian for electrons in graphene considering that electrons can hop to both nearest- and next-nearest-neighbor atoms has the form (we use units such that $\hbar=1$ ):

$$
\begin{aligned}
& H=-t \sum_{\langle i, j\rangle}\left(a_{i}^{\dagger} b_{j}+\text { H.c. }\right) \\
& -t^{\prime} \sum_{\langle\langle i, j\rangle\rangle}\left(a_{i}^{\dagger} a_{j}+b_{i}^{\dagger} b_{j}+\text { H.c. }\right),
\end{aligned}
$$

where $a_{i, \sigma}\left(a_{i, \sigma}^{\dagger}\right)$ annihilates (creates) an electron with spin $\sigma(\sigma=\uparrow, \downarrow)$ on site $R_{i}$ on sublattice A (an equivalent definition is used for sublattice B), $t$ $(\approx 2.8 \mathrm{eV})$ [13] is the nearest-neighbor hopping energy (hopping between different sublattices), and $t^{\prime}$ is the next nearest-neighbor hopping energy (hopping in the 
same sublattice) [13]. The value of $t^{\prime}$ is not well known but $a b$ initio calculations [6] find $0.02 t \lesssim t^{\prime} \lesssim 0.2 t$ depending on the tight-binding parameterization. By means of the Fourier transformation on operator the Hamiltonian is $H=-t \sum_{i}\left[a(k)^{\dagger} b(k) \exp \left(\mathrm{i} k \delta_{i}\right)+\right.$ $\left.b(k)^{\dagger} a(k) \exp \left(-\mathrm{i} k \delta_{i}\right)\right]+t^{\prime} \sum_{i}\left[a(k)^{\dagger} a(k)\left(\exp \left(\mathrm{i} k \eta_{i}\right)+\right.\right.$ $\left.\exp \left(-\mathrm{i} k \eta_{i}\right)\right]+b(k)^{\dagger} b(k)\left[\exp \left(\mathrm{i} k \eta_{i}\right)+\exp \left(-\mathrm{i} k \eta_{i}\right)\right]$. By definition of $f(k)=-t\left(\exp \left(\mathrm{i} k a_{1}\right)+\exp \left(\mathrm{i} k a_{2}\right)+1\right)$ and $s(k)=\cos \left(k a_{1}\right)+\cos \left(k a_{2}\right)+\cos \left(k\left(a_{2}-a_{1}\right)\right)$ and relation between $a$ and $\delta$ the Hamiltonian have the form

$$
H=\left(\begin{array}{ll}
a(k)^{\dagger} & b(k)^{\dagger}
\end{array}\right)\left(\begin{array}{cc}
t^{\dagger} s(k) & t f(k) \\
t f(-k) & t^{\dagger} s(k)
\end{array}\right)\left(\begin{array}{c}
a(k) \\
b(k)
\end{array}\right) .
$$

The energy bands derived from this Hamiltonian are obtained by diagonalization of Hamiltonian by transformation matrix $S$, so

$$
H=\left(\begin{array}{ll}
a(k)^{\dagger} & b(k)^{\dagger}
\end{array}\right) S S^{-1} H S S^{-1}\left(\begin{array}{c}
a(k) \\
b(k)
\end{array}\right) .
$$

$S$ can be found by some calculation

$$
\begin{aligned}
& S=\left(\begin{array}{cc}
\sqrt{\frac{f(k)}{f(-k)}} & -\sqrt{\frac{f(k)}{f(-k)}} \\
1 & 1
\end{array}\right), \\
& S^{-1}=1 / 2\left(\begin{array}{cc}
\sqrt{\frac{f(-k)}{f(k)}} & 1 \\
-\sqrt{\frac{f(-k)}{f(k)}} & 1
\end{array}\right) .
\end{aligned}
$$

Diagonalized Hamiltonian has the form

$$
\begin{aligned}
& H_{\mathrm{d}}= \\
& \left(\begin{array}{cc}
t^{\prime} s(k)+t \sqrt{f(k) f(-k)} & 0 \\
0 & t^{\prime} s(k)-t \sqrt{f(k) f(-k)}
\end{array}\right) .
\end{aligned}
$$

Energy dispersion relations are

$$
\begin{aligned}
& E_{1}=t^{\prime} s(k)+t \sqrt{f(k) f(-k)}, \\
& E_{2}=t^{\prime} s(k)-t \sqrt{f(k) f(-k)} .
\end{aligned}
$$

In this stage by choosing (2.5), (2.6) for upper and lower energy band, we show the full band structure of graphene with both $t^{\prime}$ and $t$ in Fig. 2 .

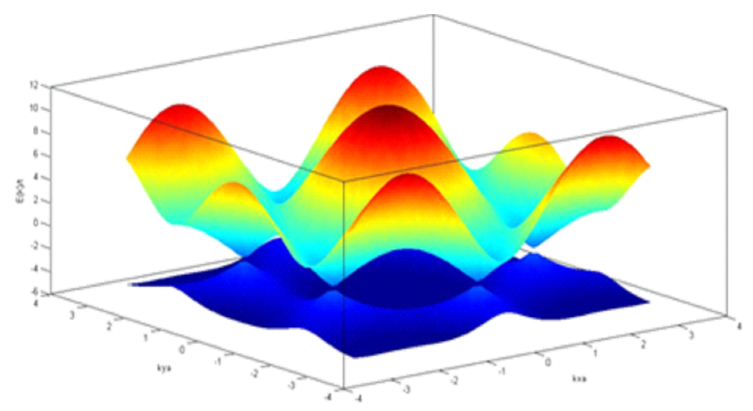

Fig. 2. Electronic dispersion in honeycomb lattice.

\section{Berry curvature throughout Brillouin zone}

In the next step we focused on calculation of the Berry curvature. Now, let us define the Berry connection in the following way:

$$
A_{\boldsymbol{k}}=\sum_{\text {occupied state }}\left\langle\psi\left|\mathrm{i} \partial_{k}\right| \psi\right\rangle .
$$

The Berry connection implies the Berry curvature as follows [14]:

$$
\Omega_{k_{x}, k_{y}}=\partial_{k_{x}} A_{k_{y}}-\partial_{k_{y}} A_{k_{x}} \text {. }
$$

So finding the eigenstates of Hamiltonian in new state is necessary. The total function for $c_{1}(k)^{\dagger}|0\rangle$ is linear combination of $\left|\varphi_{\mathrm{A}}\right\rangle$ that located in position of carbons $\mathrm{A}$ and $\left|\varphi_{\mathrm{B}}\right\rangle$ that located in position of carbons $\mathrm{B}$. Also for $c_{2}(k)^{\dagger}\left|\varphi_{\mathrm{A}}\right\rangle=\frac{1}{\sqrt{N}} \sum_{R} \mathrm{e}^{\mathrm{i} k R}\left|p_{z}, R\right\rangle,\left|\varphi_{\mathrm{B}}\right\rangle=$ $\frac{1}{\sqrt{N}} \sum_{R} \mathrm{e}^{\mathrm{i} k R}\left|p_{z}, R-d\right\rangle$. The $p_{z}$ atomic orbitals are oriented perpendicular to the plane and are rotational symmetric around the $z$-axis. By

$$
\begin{aligned}
& c_{1}(k)^{\dagger}=\sqrt{\frac{f(k)}{f(-k)}} a(k)^{\dagger}+b(k)^{\dagger}, \\
& c_{2}(k)^{\dagger}=-\sqrt{\frac{f(k)}{f(-k)}} a(k)^{\dagger}+b(k)^{\dagger} .
\end{aligned}
$$

We find $|\psi\rangle$ as

$$
|\psi\rangle=\frac{1}{\sqrt{N}} \sum_{R} \mathrm{e}^{\mathrm{i} k R}\left(\sqrt{\frac{f(k)}{f(-k)}}\left|p_{z}, R\right\rangle+\left|p_{z}, R-d\right\rangle\right) .
$$

By finding eigenstates (3.5) and replacing on the Berry curvature Eq. (3.2) we have $\Omega_{k_{x}, k_{y}}=0$.

\section{Breaking symmetry and Berry phase in Dirac points}

By consideration of Eq. (2.1), low-energy description near the Dirac points can be written as

$$
H=v\left(\xi q_{x} \sigma_{x}+q_{y} \sigma_{y}\right)-3 t^{\prime} I,
$$

where $v=\frac{\sqrt{3}}{2} a t \approx 10^{6} \mathrm{~m} / \mathrm{s}$ is the Fermi velocity, $\left(\sigma_{x}, \sigma_{y}\right)$ are the Pauli matrices and $\xi= \pm 1$, for one $K$ point (e.g. $\xi=+1$ ) we have a 2 component wave function that called pseudo spinor wave function (massless Dirac fermions),

$$
\Psi=\frac{1}{\sqrt{2}}( \pm) 1 \mathrm{e}^{\mathrm{i} \theta_{q}},
$$

where $\theta_{q}=\tan ^{-1}\left(q_{y} / q_{x}\right)$. With replacement in Eq. (3.1) the connection is acquired

$$
\begin{aligned}
& A_{q_{y}}=\frac{-q_{x}}{2\left(q_{y}^{2}+q_{x}^{2}\right)}, \\
& A_{q_{x}}=\frac{q_{y}}{2\left(q_{y}^{2}+q_{x}^{2}\right)},
\end{aligned}
$$

and by Eqs. (3.2), (4.2), (4.3) the Berry curvature is equal 


$$
\begin{aligned}
\partial_{q_{x}} A_{q_{y}} & =\frac{-2 q_{y}^{2}}{4\left(q_{y}^{2}+q_{x}^{2}\right)^{2}}, \\
\partial_{q_{y}} A_{q_{x}} & =\frac{-2 q_{y}^{2}}{4\left(q_{y}^{2}+q_{x}^{2}\right)^{2}}, \\
\Omega_{q_{y}, q_{x}} & =\partial_{q_{y}} A_{q_{x}}-\partial_{q_{x}} A_{q_{y}}=0 .
\end{aligned}
$$

But the Berry phase is

$$
\varphi_{\text {Berry }}=\oint_{0}^{2 \pi}\left\langle\Psi\left|\mathrm{i} \partial_{\theta}\right| \Psi\right\rangle \mathrm{d} \theta= \pm \pi .
$$

The rotation in $k$ space by $2 \pi$ leads to the change in the phase by $\pi$, i.e., a sign change. In these models where nearest neighbor hopping or next nearest neighbor hopping are taken into account, there is still an accidental degeneracy that makes the particle-like band of one flavor to be degenerate with the antiparticle-like (hole-like) band of the other flavor at the $K\left(K^{\prime}\right)$ point in each valley. This degeneracy can be easily lifted by the application of a perpendicular electric field that breaks the inversion symmetry in the system [13]. In graphene, the quantum Berry phase in the presence of magnetic field leads to anomalous half-integer quantum Hall effect [2, 15].

Next by adding the field (graphene on substrate), we reviewed its effects on the Berry curvature of the Dirac points. In graphene the $\mathrm{A}$ sites are identical to the $\mathrm{B}$ sites modulo a $\pi$ rotation, so the energies $\mathcal{E}_{\mathrm{A}}=\mathcal{E}_{\mathrm{B}}[16]$. But as detailed in Ref. [17], we allow for different site energies $\left(\mathcal{E}_{\mathrm{A}} \neq \mathcal{E}_{\mathrm{B}}\right)$, as in hexagonal boron nitride, and take the graphene limit where it is appropriate. So the Hamiltonian has a form

$$
\begin{aligned}
H & =\sum_{i}\left(\mathcal{E}_{\mathrm{A}} a_{i}^{\dagger} a_{i}+\mathcal{E}_{\mathrm{B}} b_{i}^{\dagger} b_{i}\right)-t \sum_{\langle i, j\rangle}\left(a_{i}^{\dagger} b_{j}+\text { H.c. }\right) \\
& -t^{\prime} \sum_{\langle\langle i, j\rangle\rangle}\left(a_{i}^{\dagger} a_{j}+b_{i}^{\dagger} b_{j}+\text { H.c. }\right) .
\end{aligned}
$$

Here the energy difference $\Delta$ is defined by $\Delta \equiv \frac{\mathcal{E}_{\mathrm{A}}-\mathcal{E}_{\mathrm{B}}}{2}$, and the energy origin is chosen such that $\frac{\mathcal{E}_{\mathrm{A}}+\mathcal{E}_{\mathrm{B}}}{2}=0$. For relatively low doping, we can resort to the low-energy description near the Dirac points. The Hamiltonian is given by $[17,18]$ :

$$
H=v\left(\xi q_{x} \sigma_{x}+q_{y} \sigma_{y}\right)+\frac{\Delta}{2} \sigma_{z}-3 t^{\prime} I,
$$

where $\sigma$ is the Pauli matrix accounting for the sublattice index, and $q$ is measured from the valley center $K_{1,2}=$ $( \pm 4 \pi / 3 a) \hat{x}$ with $a$ being the lattice constant. The Berry curvature of the conduction band is given by [18]:

$$
\Omega_{q_{x}, q_{y}}=\xi \frac{3 a^{2} t^{2} \Delta}{2\left(\Delta^{2}+3 q^{2} t^{2} a^{2}\right)^{3} / 2} .
$$

We remark that as $\Delta$ goes to zero, the Berry curvature vanishes everywhere except at the Dirac points where it diverges.

\section{Discussion and conclusion}

Energy band structure that was obtained by Wallace [10] was $E_{\mp}(k)=\mp t \sqrt{3+g(k)}-t^{\prime} g(k)$ with specific $g(k)$.
But in our calculation we diagonalized Hamiltonian and plotted it in Fig. 2. In resumption the Berry curvature of these states has been calculated and got zero. Our assumption was that we do not know two symmetries of graphene, calculated from eigenstates. Our result shows that the next nearest neighborhood hopping did not break the symmetry of system. We know if the system has time reversal symmetry, the symmetry condition requires that $\Omega(-k)=-\Omega(k)$. If the system has spatial inversion symmetry, then $\Omega(-k)=\Omega(k)$. Therefore, for crystals with simultaneous time-reversal and spatial inversion symmetry the Berry curvature vanishes identically throughout the Brillouin zone [14]. There are many important physical systems where both symmetries are not simultaneously present, for example single-layer graphene sheet with staggered sublattice potential, breaks inversion symmetry, so in this situation the Berry curvature is not zero, [19] when we just consider nearest and next nearest neighbors (without any substrate). The Berry phase around the Dirac points becomes exactly $\pm \pi$, however the integration of the Berry curvature on the Brillouin zone is zero.

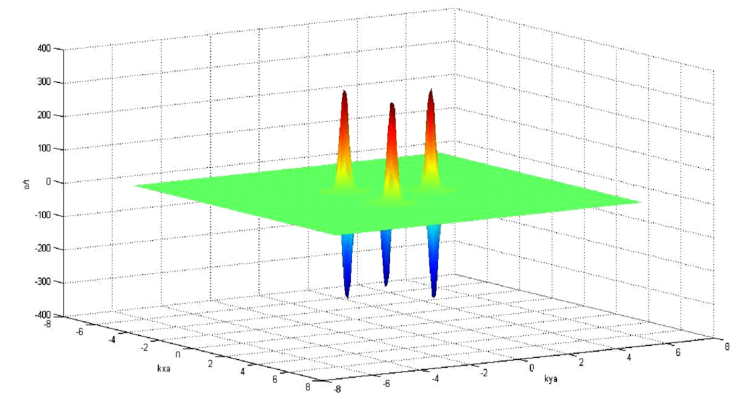

Fig. 3. Berry curvature in Brillouin zone of a graphene sheet with broken inversion symmetry.

Figure 3 shows the Berry curvature in six points of the Brillouin zone where $\Delta=0.28 \mathrm{eV}$ [14]; as is seen, the Berry curvatures from the Dirac points can compensate each other. The $t^{\prime}$ term is isotropic around $K$. The most salient feature of this term is that it is exactly symmetric between the $\mathrm{A}$ and $\mathrm{B}$ sublattices (as one might perhaps anticipate from its sixfold symmetry); hence, it gives a contribution $-t^{\prime} f(k)$, which is proportional, in the "spinor" representation, to the unit matrix. Such a term destroys the perfect symmetry of the band around $\epsilon=0$ (since the term $-t^{\prime} f(k)$ is added to both the upper and the lower branches); however, it cannot spoil the degeneracy of the two solutions at the Dirac points $K$ and $K^{\prime}$.

\section{References}

[1] K.S. Novoselov, A.K. Geim, S.V. Morozov, D. Jiang, Y. Zhang, S.V. Dubonos, I.V. Grigorieva, A.A. Firsov, Science 306, 666 (2004). 
[2] K.S. Novoselov, A.K. Geim, S.V. Morozov, D. Jiang, M.I. Katsnelson, I.V. Grigorieva, S.V. Dubonos, A.A. Firsov, Nature (London) 438, 197 (2005).

[3] D.S.L. Abergel, A. Russell, V.I. Fal'ko, Appl. Phys. Lett. 91, 063125 (2007).

[4] A.K. Geim, K.S. Novoselov, Nature Mater. 6, 183 (2007).

[5] T.W. Odom, J.L. Huang, P. Kim, C.M. Lieber, J. Phys. Chem. B 104, 2794 (2000).

[6] S. Reich, J. Maultzsch, C. Thomsen, P. Ordejon, Phys. Rev. B 66, 035412 (2002).

[7] V.N. Popov, L. Henrard, Phys. Rev. B 70, 115407 (2004).

[8] B. Partoens, F.M. Peeters, Phys. Rev. B 74, 075404 (2006).

[9] B. Partoens, F.M. Peeters, Phys. Rev. B 75, 193402 (2007).

[10] P.R. Wallace, Phys. Rev. 71, 622 (1947).

[11] R. Saito, G. Dresselhaus, M.S. Dresselhaus, Physical Properties of Carbon Nanotubes, Imperial, London 1998 , p. 26
[12] M.V. Berry, Proc. R. Soc. Lond. A 392, 45 (1984).

[13] A.H. Castro Neto, F. Guinea, N.M.R. Peres, K.S. Novoselov, A.K. Geim, Rev. Mod. Phys. 81, 109 (2009).

[14] D. Xiao, M. Chang, Q. Niu, Rev. Mod. Phys. 82, 1959 (2010).

[15] Y. Zhang, Y.-W. Tan, H.L. Stormer, P. Kim, Nature 438, 201 (2005).

[16] M. Mecklenburg, B.C. Regan, Phys. Rev. Lett. 106 , 116803 (2011).

[17] G.W. Semenoff, Phys. Rev. Lett. 53, 2449 (1984).

[18] D. Xiao, W. Yao, Q. Niu, Phys. Rev. Lett. 99, 236809 (2007).

[19] S.Y. Zhou, G.-H. Gweon, A.V. Fedorov, P.N. First, W.A. de Heer, D.-H. Lee, F. Guinea, A.H.C. Neto, A. Lanzara, Nature Mater. 6, 770 (2007). 